OPEN Mechanisms of recovery of visual

SUBJECT AREAS:

NEUROSCIENCE

PATTERN VISION

Received

31 July 2014

Accepted

24 December 2014

Published

26 February 2015

Correspondence and requests for materials should be addressed to D.M.L. (dlevi@ berkeley.edu)

* These authors contributed equally to this work. \section{function in adult amblyopia through a tailored action video game}

\author{
Indu Vedamurthy ${ }^{1 *}$, Mor Nahum ${ }^{2 *}$, Daphne Bavelier ${ }^{1,3}$ \& Dennis M. Levi ${ }^{2}$
}

\author{
'Department of Brain \& Cognitive Sciences, University of Rochester. Rochester, New York 14627-0268, U.S.A, ${ }^{2}$ School of \\ Optometry, Graduate Group in Vision Science and Helen Wills Neuroscience Institute, University of California, Berkeley. Berkeley, \\ California, 94720-2020, ${ }^{3}$ Faculty of Psychology and Education Sciences, University of Geneva, Switzerland.
}

Amblyopia is a deficit in vision that arises from abnormal visual experience early in life. It was long thought to develop into a permanent deficit, unless properly treated before the end of the sensitive period for visual recovery. However, a number of studies now suggest that adults with long-standing amblyopia may at least partially recover visual acuity and stereopsis following perceptual training. Eliminating or reducing interocular suppression has been hypothesized to be at the root of these changes. Here we show that playing a novel dichoptic video game indeed results in reduced suppression, improved visual acuity and, in some cases, improved stereopsis. Our relatively large cohort of adults with amblyopia, allowed us, for the first time, to assess the link between visual function recovery and reduction in suppression. Surprisingly, no significant correlation was found between decreased suppression and improved visual function. This finding challenges the prevailing view and suggests that while dichoptic training improves visual acuity and stereopsis in adult amblyopia, reduced suppression is unlikely to be at the root of visual recovery. These results are discussed in the context of their implication on recovery of amblyopia in adults.

A mblyopia is a deficit in vision that arises from abnormal visual experience early in life. Although the nature of this visual deficit is not fully understood, it is thought that interocular suppression, or inhibition of the amblyopic eye by the strong eye, is a feature, and possibly a cause, of amblyopia ${ }^{4}$, and there is ample clinical $^{5}$, psychophysical ${ }^{6-16}$ and physiological ${ }^{17-19}$ evidence for this view point.

Traditionally, amblyopia was thought to develop into a permanent deficit, unless properly treated before the end of the sensitive period for visual recovery ${ }^{4}$. In line with this view, patching of the strong eye has been the predominant form of treatment for amblyopia in children since the eighteenth century ${ }^{20,21}$, and was considered ineffective beyond the age of seven ${ }^{4}$. However, a number of studies now suggest that adults with long-standing amblyopia may at least partially recover visual acuity and stereopsis following monocular perceptual training ${ }^{1}$ or video game play with their amblyopic eye ${ }^{2,3}$. Moreover, a number of recent studies in children ${ }^{22-24}$ and adults $\mathrm{s}^{25-30}$ point to a reduction of suppression through dichoptic training as the key to successful treatment. Still, both the link between suppression and amblyopia ${ }^{31,32}$ and the safety and efficacy of dichoptic treatment ${ }^{33}$ are controversial.

The current study aimed at shedding light on the connection between reduced suppression and improved visual function following treatment in adult amblyopia. Twenty-three adults with amblyopia, ten with anisometropia (unequal refractive error) and thirteen with strabismus (a turned eye), played 40 hours of a customized dichoptic action video game. This customized game was designed to reduce suppression, promote fusion and increase attention by the amblyopic eye under binocular conditions. These design goals were accomplished by presenting identical images to the two eyes, with the perceptual strength of the image seen by the strong eye decreased to perceptually match that of the weak (amblyopic) eye (AE), and by incorporating a perceptual learning task exclusively targeting the amblyopic eye [see Methods for full details]. Reducing the luminance/ contrast is an effective method for balancing the input to the two eyes ${ }^{11,15,34-36}$. In addition, we performed frequent "suppression checks" to ensure successful fusion. The dichoptic video game was viewed through a stereoscope. The sizeable number of participants in our study allowed us, for the first time in the literature, to assess the link between visual function recovery and suppression. Surprisingly, we found that decreased suppression was not significantly correlated with improved vision. This suggests that reduced suppression is unlikely to be at the root of visual recovery. 

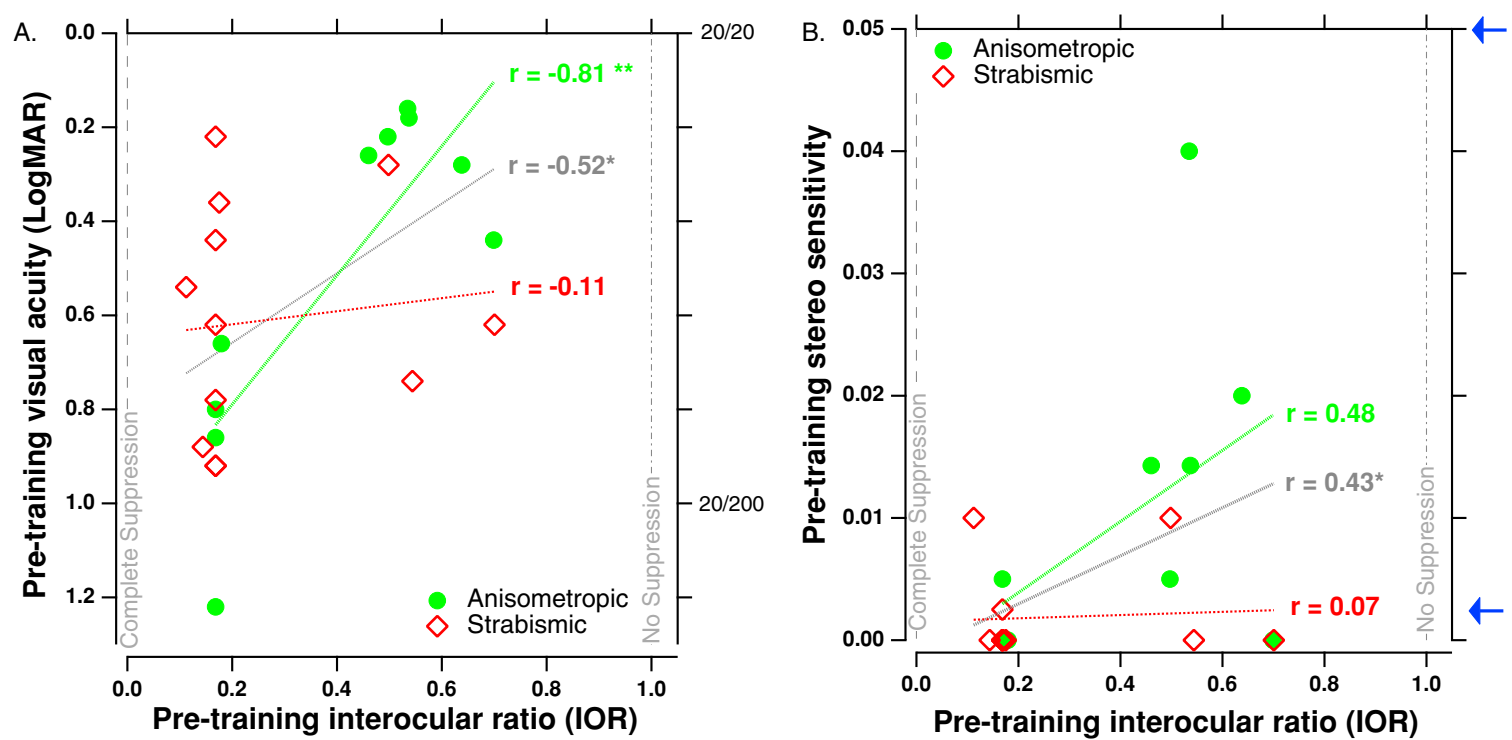

Figure 1 Pre-training (baseline) correlations with interocular suppression. (A). The relationship between pre-training IOR and pre-training AE visual acuity. Green symbols and red symbols show data for anisometropic and strabismic amblyopes respectively. The lines show linear regression fits to the data (green - anisometropic; red - strabismic; gray -all amblyopes). The gray vertical lines at abscissa values of 0 and 1 represent complete suppression and no suppression respectively. (B). The relationship between pre-training IOR and pre-training stereo sensitivity (1/stereo threshold in arc sec). Note that many strabismic amblyopes had no stereovision prior to training, hence some of the red symbols overlap on the figure. Blue arrows along the ordinate show the upper and lower limits of the test at 20 (top arrow) and $400 \operatorname{arc~sec}$ (lower arrow) respectively. The asterisks indicate significance level. * $=\mathrm{p}<0.05 ; * *=\mathrm{p}<0.01$.

\section{Results}

Interocular Ratio as an index for suppression. At the beginning of each session, subjects carefully aligned the stereoscope and reduced the perceptual strength of the strong eye's image (by adjusting the alpha value, see Methods for details) relative to the amblyopic eye's image to perceptually equalize the input to the two eyes. The interocular Ratio (IOR - the ratio of strong eye to AE luminance/ contrast) provides a convenient index for suppression ${ }^{15}$, with higher ratios indicating less suppression. IOR of 0 indicates complete suppression while IOR of 1 indicates no suppression.
Prior to training, for the group-as-a-whole, IOR was significantly correlated with both the baseline clinical visual acuity (specified as LogMAR; Fig. 1A) and stereo sensitivity (1/stereo threshold in arc sec; Fig. 1B). Table 1 shows the correlation coefficients and p-values for the group and also separately for anisometropic (green symbols throughout) and strabismic (red symbols throughout) amblyopes. It is interesting to note that prior to training, anisometropic amblyopes (green symbols) show strong correlations between visual function and IOR, whereas strabismic amblyopes (red symbols) show effectively no correlation.

Table 1 | Pre-training correlations

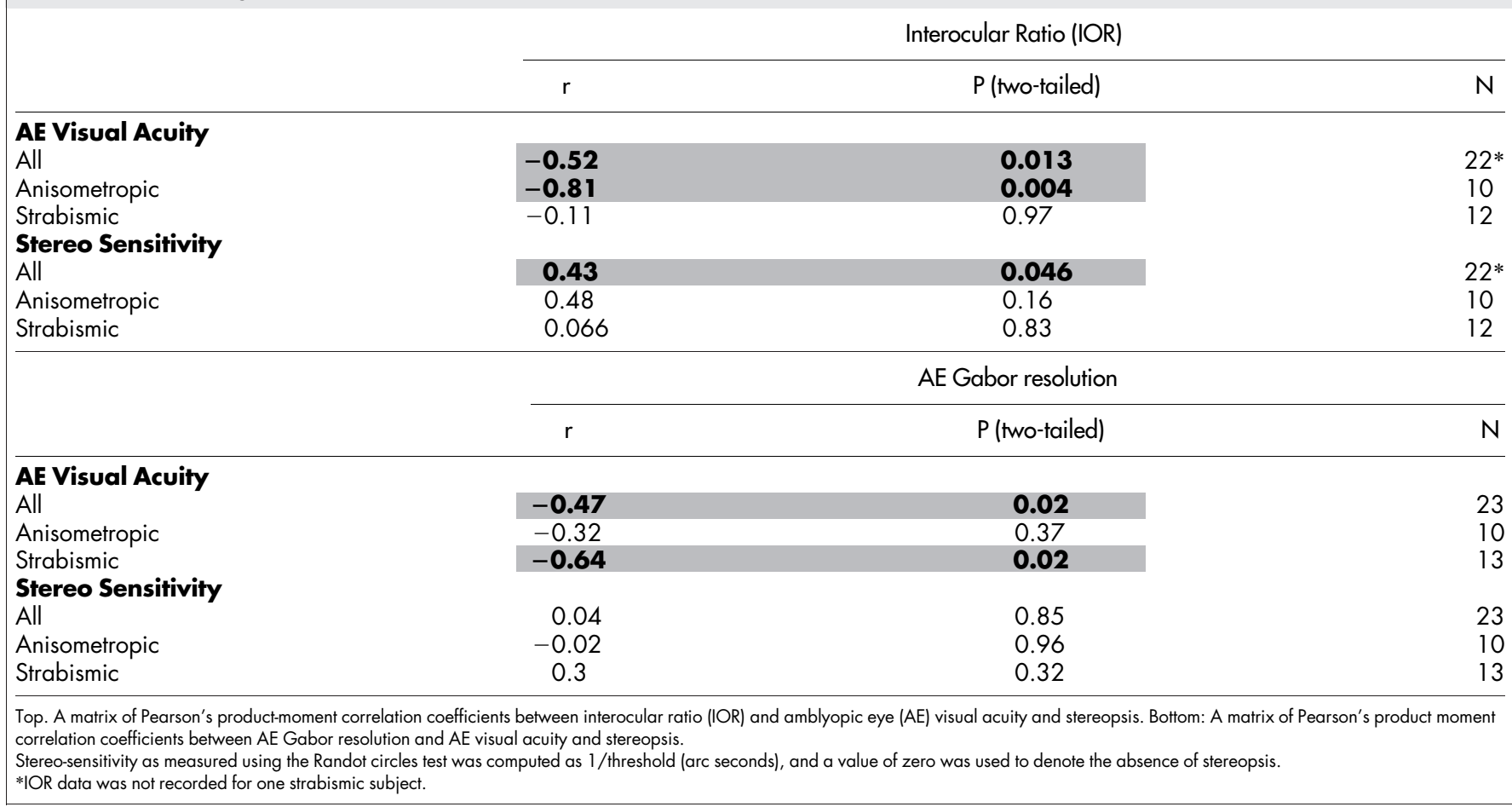


A.

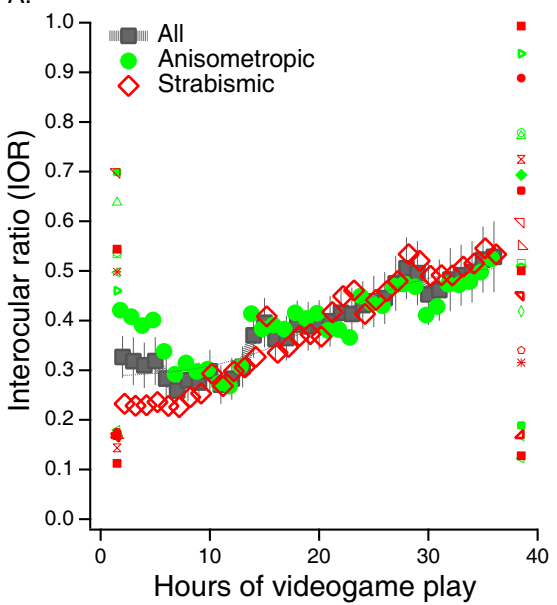

B.

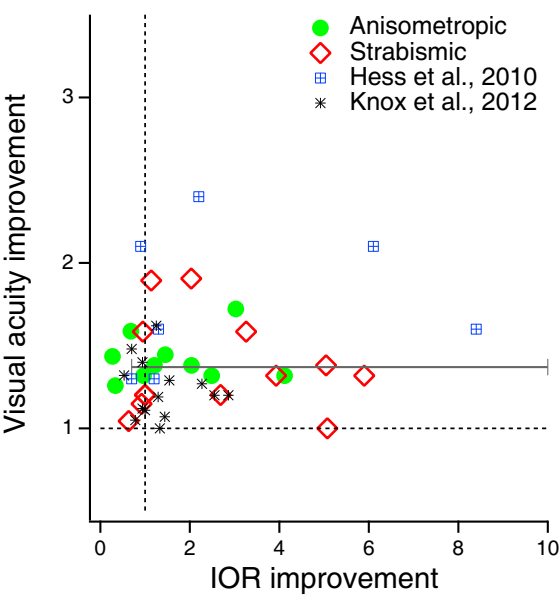

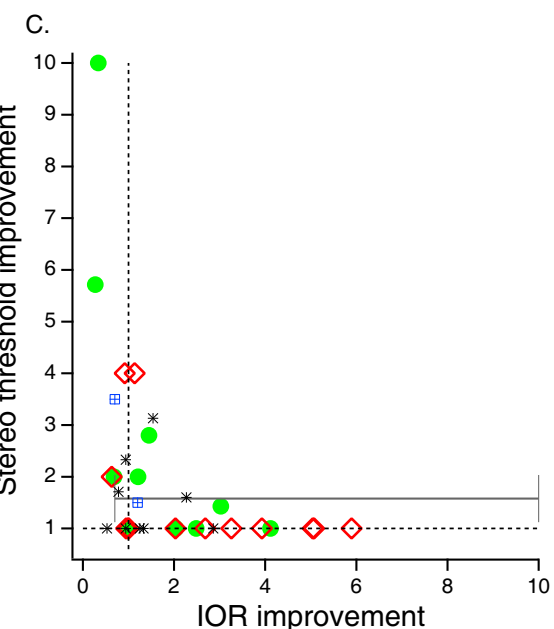

Figure $2 \mid$ Video game training induced reduction in interocular suppression (as measured by IOR). (A). IOR as a function of video game training hours. Squares show mean data for all participants $( \pm 1$ SEM). Large green circles and red diamonds show mean data for anisometropic and strabismic amblyopes respectively. Small symbols represent the IOR for the first 4 and last 4 hours for each individual subject. IOR ranges from 0 (complete suppression) to 1 (no suppression). (B). Scatter plot illustrating the lack of correlation between training induced changes in visual acuity (ordinate) and suppression reduction as measured by IOR improvement (abscissa). Training-induced changes are denoted as pre versus post ratios; ratio $>1$ represents improved performance following training; thin horizontal and vertical lines denote a ratio of 1 (i.e. no change in performance). Blue squares (with cross) and black asterisks show data re-plotted from other video game training studies [Hess et al., 2010, see Ref. 25; and Knox et al., 2012, see Ref. 22 for comparison]. (C). Scatter plot illustrating an unexpected negative correlation between training induced improvements in stereopsis and reduction in suppression (denoted by IOR improvement). The horizontal line segments in B and C show the mean improvement \pm 1 SEM.

Interocular Suppression is reduced over the course of training. During the course of game-play, IOR increased, on average, by a factor of $\approx 1.6$ (Fig. $2 \mathrm{~A}$, gray dotted line, see also Table 2), indicating a significant reduction in suppression. However, there was substantial individual variation: for example, some participants showed an increase in ratio (decrease in suppression), from a ratio of 0.17 (AE luminance/contrast roughly 6 times that of the strong eye) to close to 1.0 (no suppression), while others showed no change, or even a small decrease in ratio (Fig. 2A).

We measured visual acuity and stereoacuity before and after the course of game play [see Methods]. Following the training period, all but one of our amblyopic participants showed improvements in visual acuity (by, on average, a factor of 1.4 ), with seventeen participants $(74 \%)$ showing one or more lines of improvement on the letter chart. Nine participants (39\%) improved in stereopsis, one from 200 to 20 arc sec (a factor of 10 ).

Fig. 1 and Table 1 show that at baseline, poorer AE visual acuity and stereo sensitivity are linked with greater magnitudes of suppression. This is especially the case for anisometropic amblyopes. As noted above, several recent studies have suggested that reducing suppression may be the key to improving visual acuity and stereopsis in adults with long-standing amblyopia ${ }^{25-30}$. Thus, given the large individual variation, we would expect that the degree of reduction in suppression would be correlated with the degree of improvement in visual acuity and/or in stereopsis following training. However, as can be seen in Fig. 2, and Table 3, this is not the case. Although visual acuity improved and suppression was reduced following training, the improvement in visual acuity was not significantly correlated with the change in IOR (Fig. 2B), and changes in suppression accounted for less than 2 percent of the variance in visual acuity improvement. Moreover, we found a surprising negative correlation between training-induced improvements in stereopsis and suppression reduction as measured by IOR $(\mathrm{r}=-0.47, \mathrm{p}<0.05)$. This is driven largely by the three anisometropic amblyopes who showed the largest improvements in stereo acuity, but with little change in IOR.

Results are similar even if we limit the analysis to include only those participants who had measurable stereopsis prior to training: there is no correlation between improvements in visual acuity/stereopsis and reduced suppression, nor is there a significant correlation between the baseline suppression level and the improvement in visual acuity (Supplemental Fig. S1A). On the other hand, we do find a significant correlation $(0.496 ; \mathrm{p}=0.019)$ between the initial IOR level, and the improvement in stereopsis (Supplemental Fig. S1B). This correlation is derived primarily from the fact that the two anisometropic amblyopes with the largest improvement in stereopsis, had the least initial suppression.

Gabor resolution by the amblyopic eye under binocular conditions. Under normal binocular viewing conditions, the weak, noisy, and unreliable signals coming from the amblyopic eye may be ignored, suppressed or unresolved. An adaptive Gabor discrimination task, similar to the ones employed in perceptual learning studies, was presented to the AE during game play, with the spatial frequency of the Gabor target adaptively changed in order to maintain performance

Table 2 | Pre- and Post-Training Assessment of Visual Function

\begin{tabular}{lccc} 
Visual function & Pre-training mean $\pm 1 S D$ & Post-training mean \pm 1SD & Pre-post comparison (t-value) \\
\hline IOR & $0.33 \pm 0.22$ & $0.52 \pm 0.27$ & $-2.64^{\star}$ \\
Gabor resolution acuity (cpd) & $2.51 \pm 1.28$ & $5.58 \pm 2.67$ & $-6.87^{\star \star}$ \\
Visual acuity (LogMAR) & $0.58 \pm 0.30$ & $0.44 \pm 0.29$ & $9.12^{\star \star}$ \\
Stereo-sensitivity (1/arc seconds) & $0.005 \pm 0.01$ & $0.012 \pm 0.017$ & $-2.54^{\star}$ \\
\hline
\end{tabular}

Mean ( \pm 1SD) data are shown. Pre-post comparison (last column) was done using a two-tailed paired samples t-tests ( $\mathrm{p}<0.05$. $\star \star p<0.01$ ). Stereo-sensitivity was computed as $1 /$ threshold (arc seconds), and a value of zero was used to denote the absence of stereopsis. Sample size $n=23$ for each measure except for IOR ( $n=22$ ). LogMAR, logarithm of the minimum angle of resolution; cpd, cycles per degree; $A E$, amblyopic eye. 
Table 3 | Pre versus Post-Training Ratio correlations

\begin{tabular}{lcccc} 
Visual function & IOR & GaborResolution in game (cpd) & Visual acuity (LogMAR) & Stereopsis(arc sec) \\
\hline IOR & - & 0.03 & -0.13 & $-0.47^{*}$ \\
Gabor Resolution in game (cpd) & - & & 0.04 & 0.11 \\
Visual acuity (LogMAR) & & - & 0.00 \\
\hline
\end{tabular}

A matrix of Pearson's product-moment correlation coefficients between the four ratiovariables-IOR, Gabor resolution acuity (AE), visual acuity (AE), and stereopsis. For each visual function, ratio larger than 1 represents improvement in performance following video game play, while ratio of 1 represent no change from baseline, and ratio $<1$ denotes deterioration in performance. For the stereopsis test (Randot circles test), a threshold value of 600 arc seconds was assigned if participants failed the test. Correlations were performed on the ratios. One Star indicates two-tailed statistical significance ( $\left.{ }^{*} p<0.05\right)$. Sample size $n=23$ for each measure except for IOR ( $n=22$ ). LogMAR, logarithm of the minimum angle of resolution; cpd, cycles per degree; $A E$, amblyopic eye.

at the $79 \%$ correct level [see Methods]. While this is not a standard measure of suppression, the resolution of a Gabor target embedded into the game provides a measure of the AE's resolution acuity under dichoptic viewing conditions.

Not surprisingly, prior to training, Gabor resolution was significantly correlated with initial AE visual acuity for the group-as-awhole (see Fig. 3A and Table 1, bottom). However, no such correlation was found with baseline stereo sensitivity (1/stereo threshold in arc sec; Fig. 3B).

Similar to the IOR, the Gabor target's resolution in AE increased by a factor of $\approx 2.6$ on average, indicating increased resolution acuity of the amblyopic eye under dichoptic viewing conditions, but with large individual variations (Fig. 4A). Of note, there was no correlation between the increase in IOR and the increase in Gabor resolution acuity of the AE following training (Table 3), suggesting that these two measures are tapping into different aspects of amblyopic vision: while IOR measures how well input from the strong eye can be tolerated while still allowing fusion, the resolution of Gabor targets measures how well the amblyopic eye can resolve a target under conditions of binocular fusion.

As with IOR, there was no significant correlation between the change in Gabor resolution and improvements in either visual acuity (Fig. 4B) or stereoacuity (Fig. 4C) after game training (see also Table 3); nor is there a significant correlation between the initial absolute Gabor resolution level, and the improvement in visual acuity or stereopsis (Supplemental Fig. S1C and D).

While Table 3 is based on the entire sample of amblyopes, considering anisometropic and strabismic amblyopes separately or just those with stereopsis prior to training also produced no significant correlations.

\section{Discussion}

Playing a highly engaging, action-packed dichoptic first-person shooter video game, customized to incorporate a perceptual learning task and perceptually equated stimuli to the two eyes, results in reduced suppression, increased Gabor resolution, improved visual acuity and, in some cases, improved stereopsis in adults with long-standing amblyopia. The improvements we found here in both visual acuity and stereopsis are consistent with those reported in many previous studies of both monocular ${ }^{1-3}$ and dichoptic ${ }^{22-30}$ perceptual learning as well as in video game play in amblyopia. However, our relatively large cohort of adults with amblyopia, allowed us, for the first time, to assess the link between visual function recovery and suppression. We found no relationship between these two variables. Given the prominence of suppression in amblyopia ${ }^{4-19}$, these results are both novel and surprising.

One potential issue is that there is not a single, standardized method used to quantify interocular suppression across studies. For example, Hess and colleagues ${ }^{25-29}$ used a dichoptic motion coherence task; Ooi and colleagues ${ }^{30}$ used a sensory dominance task. Still, a more careful look at the data from these studies reveals, albeit with a smaller sample, that there is no correlation between visual function improvement and reduction in suppression. Participants in the Hess et al. study ${ }^{25}$ (data replotted as blue squares with crosses in Fig. 2B and 2C) show a slightly larger range of improvements in both visual acuity and suppression, but there is no significant correlation between the two measures $\left(r^{2}=0.008\right)$ in their sample. In the Knox et al. ${ }^{22}$
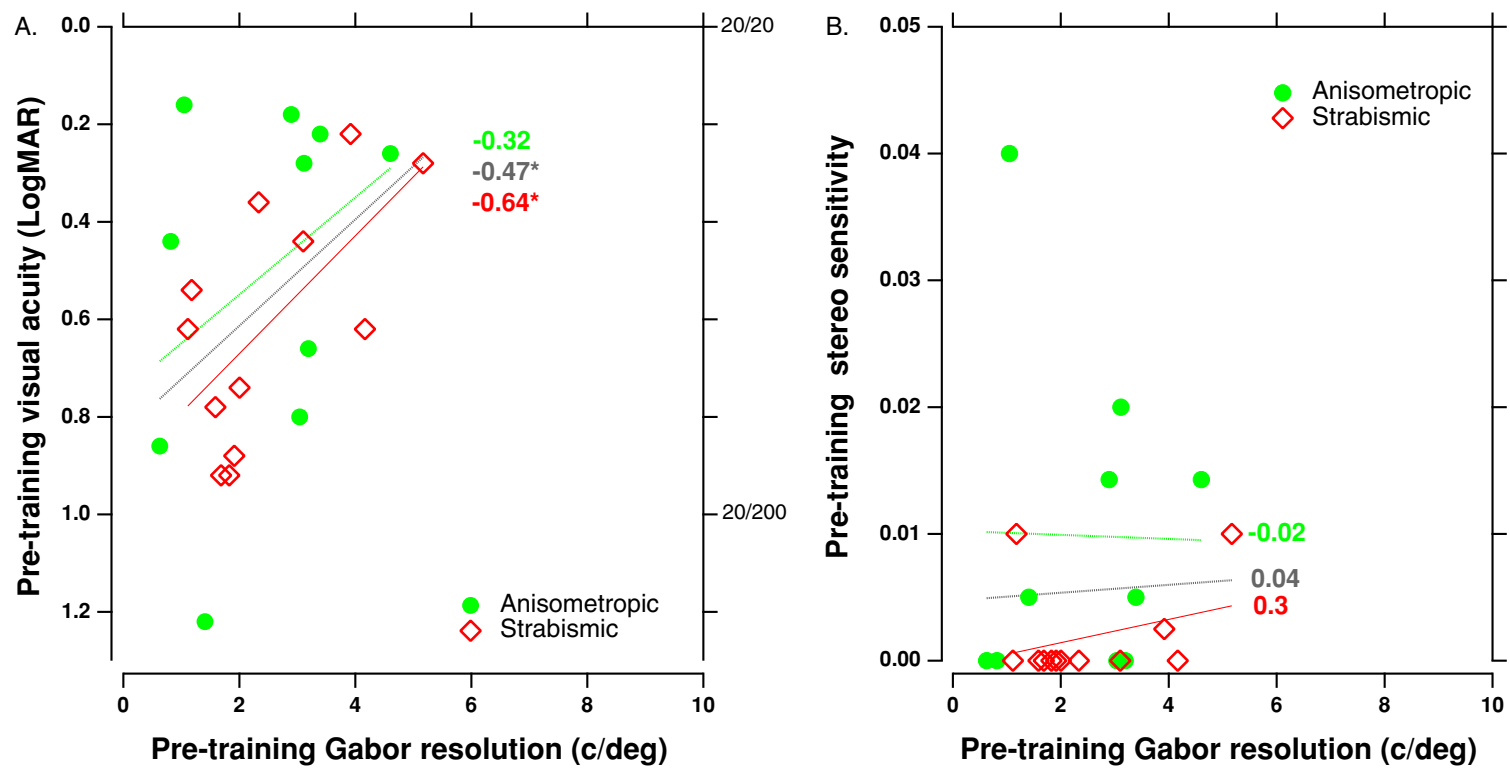

Figure 3 Pre-training correlations with AE resolution. (A). The relationship between pre-training Gabor resolution and pre-training visual acuity. Green circles and red diamond symbols show data for anisometropic and strabismic amblyopes, respectively. The lines show linear regression fits to the data (green - anisometropic; red - strabismic; gray - all amblyopes). (B). The lack of significant relationship between pre-training Gabor resolution and stereo sensitivity (1/stereo threshold in arc sec) is depicted. 

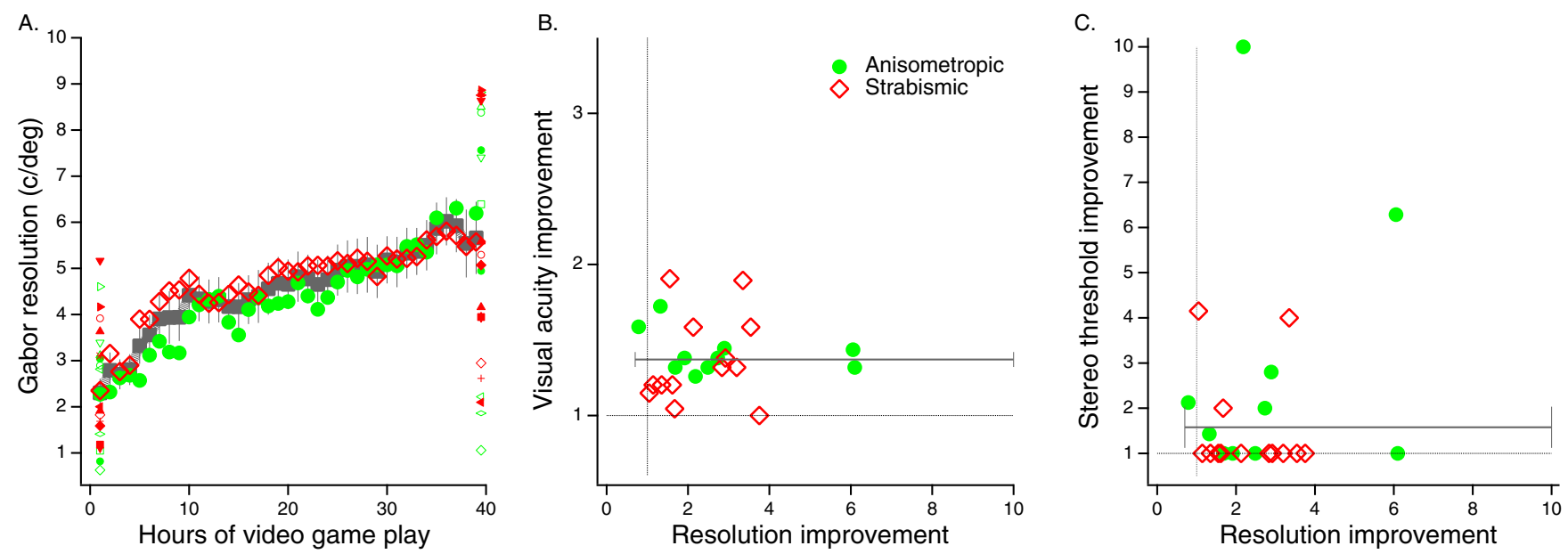

Figure $4 \mid$ (A). AE resolution acuity measured with Gabor targets as a function of video game training hours. Inter-individual variations are depicted similar to Fig. 2A. (B). Scatter plot illustrating a lack of correlation between training induced changes in AE visual acuity and Gabor resolution. (C). Scatter plot illustrating a lack of correlation between training-induced changes in stereopsis and Gabor resolution $(r=0.11, p>0.05)$. The horizontal line segments in panels $\mathrm{B}$ and $\mathrm{C}$ show the mean improvement \pm 1 SEM.

study, child participants played a Tetris-like game under dichoptic conditions, in which the interocular luminance/contrast ratio was varied, similar to our study. While their participants showed a somewhat smaller range of improvements, on a subject-by-subject basis, their data show no correlation between the reduction of suppression (as measured by the interocular ratio) and the improvements in visual acuity, or with a clinical measure of suppression (data replotted as black asterisks in Fig. 2B and 2C).

To ensure attention to the amblyopic eye during binocular game play, our novel custom video game incorporated an orientation discrimination perceptual learning task by presenting a Gabor target to the amblyopic eye, while both eyes received identical views of the perceptually balanced video game. Similar to IOR, Gabor resolution significantly improved during gameplay, but showed no correlation with improvements in visual acuity or stereo acuity. We note that the Gabor resolution is not a standard measure of suppression - rather, it provides a measure of the AE's resolution acuity under dichoptic viewing conditions. The abrupt introduction of the Gabor patch in the AE only was designed to attract attention to the amblyopic eye, which in fact may help reduce suppression, similar to the "pushpull" method ${ }^{30}$. However, we acknowledge this transient may be somewhat masked by the dynamic scene changes in the game presented dichoptically. The most important feature of our Gabor resolution task is that participants were not just to simply detect the transient, but rather had to discriminate the orientation of the grating. The transient provided no information about orientation allowing us to assess AE resolution acuity in the context of a complex visual world. Importantly, we found a substantial improvement in the amblyopic eye's resolution acuity from early to late in training, which was not correlated with the improvements in either visual acuity or stereopsis.

The extent to which the type of dichoptic training with balanced input presented here is advantageous remains unclear. A review of the extent literature indicates that perceptual learning and action video game based training (whether monocular or dichoptic) produce comparable improvements in visual acuity (typically one to two lines on an acuity chart - see Ref. 42 for a review), although training on a dichoptic Tetris-like game is more effective than playing the same game monocularly ${ }^{29}$. Moreover, monocular training has been reported to result in reduced suppression ${ }^{43}$ and improved stereopsis ${ }^{2,43-47}$ as well, mostly but not exclusively in anisometropic amblyopes. On the otherhand, dichoptic training may be more effective in promoting binocular vision and stereopsis ${ }^{22-29}$ (reviewed in Ref. 48); however it will require a randomized clinical trial in a large population of amblyopes, considering separately anisometropic and strabismic patients, to determine the most effective method for treating adults with amblyopia.

Finally, we note that the strength of interocular suppression in amblyopia depends strongly on the details of the stimulus ${ }^{6-16}$. It is noteworthy that Knox et al..$^{22}$ found no correlation between the interocular contrast ratio and a clinical measure of suppression, as is the case here for IOR. We suspect that there may be many forms of suppression, and that these may act at different neural loci ${ }^{37}$. Indeed, it has been suggested that suppression may be different in dorsal and ventral streams ${ }^{29}$. In addition, stereopsis and rivalry suppression are thought to involve independent pathways through the early stages of visual processing ${ }^{38}$. Moreover, suppression may differ in anisometropic and strabismic amblyopia. For example, there is a much stronger correlation between the degree of suppression and both visual acuity and stereopsis prior to training in anisometropic amblyopes than in strabismics (as is evident in Fig. 1 and Table 1). Indeed, anisometropic amblyopes are much more likely to have residual stereopsis and binocular vision than strabismic amblyopes ${ }^{39}$, and much less likely to suffer from crowding ${ }^{40,41}$. For these reasons, we reported the correlations for anisometropic and strabismic amblyopes separately. However, the lack of correlation between the changes in suppression and the improvement in visual performance applies to both anisometropic and strabismic amblyopes.

Thus, dichoptic training may diminish some aspects of suppression as well as improve visual acuity and/or stereopsis in some adults with amblyopia, but these may not be directly related. As exemplified in several participants reported here, significant suppression can coexist with recovery of visual acuity and/or stereopsis. To be clear, we are not arguing that reducing suppression is not important, but rather that there may be other limits, such as greater brakes on plasticity, to the recovery of visual acuity and stereopsis in adult amblyopia. A good deal of evidence, both physiological and behavioral, suggests that changing the balance of neural excitation and inhibition by reducing inhibition and boosting excitation may be crucial in recovery of visual functions ${ }^{49}$. All approaches to retraining the $\mathrm{AE}$, whether monocular video game play $^{2,3}$, perceptual learning ${ }^{1}$, the "push-pull" method ${ }^{30}$, or dichoptic training ${ }^{22-29}$ seek to achieve this altered balance by increasing signal, reducing noise, or modulating attention in the $\mathrm{AE}$. That some forms of reduced suppression ensue as a by-product of such training is clearly established, yet we show here that reduced suppression is unlikely to be at the root of visual recovery. 
above). They were then asked to adjust the luminance value of the strong eye so that

\section{Methods}

Study Participants. Twenty-three adult amblyopes, mean age: $39.57 \pm 15.74$ (range 19-62 yrs), 10 purely anisometropic, 13 strabismic or strabismic and anisometropic participated in the experiments. Subjects were unilateral amblyopes with at least a two-line difference in acuity between the eyes. Note that in the graphs and tables all subjects with strabismus (whether they have anisometropia or not) are classified as "strabismics". The Research Subjects Review Boards at the University of Rochester and the University of California, Berkeley approved the study protocol. The study was conducted according to the tenets of the Declaration of Helsinki and informed consent was obtained from each participant. The methods were carried out in accordance with the approved guidelines.

Intervention: A dichoptic custom-made Unreal Tournament video game. We have developed a dichoptic version of a commercial first-person-shooter action video game, Unreal Tournament $2004^{50}$. Previously, action games have been shown to enhance the spatial and temporal aspects of vision and visual attention ${ }^{51,52}$. The dichoptic video game combines both the highly motivating aspects of commercial action video games with the efficacy of perceptual learning. Specifically, the game is played under dichoptic viewing conditions in order to reduce suppression and promote fusion, while providing an embedded psychophysical resolution task which enables a more targeted approach to training. This custom-made game has five main innovative features, see Bayliss et al. ${ }^{53}$ for full details:

a. The game presents a split screen view, allowing independent control of the images presented to the right and left eyes.

b. The perceptual strength of the image presented to the strong eye can be adjusted to mitigate suppression and to allow fusion.

c. The game includes easier introductory levels, as well as computer-controlled game opponents. This allows individuals with little or no video game experience to gradually master the skills required to become an expert player

d. A psychophysical resolution task is embedded within the game, and is presented to the $\mathrm{AE}$ only: the user is required to discriminate the orientation of a Gabor patch and respond (i.e. shoot or ignore). An incorrect response transforms the Gabor into a game 'enemy'. The Gabor patch task enables us to monitor the AE's resolution limit under dichoptic conditions, while simultaneously serving as a suppression check, ensuring that the $\mathrm{AE}$ is actively engaged in the game.

e. To allow for fusion, the game includes nonius lines for binocular alignment, as well as 'suppression check' screens.

A video of an early version of the game, which was developed in collaboration with Jessica Bayliss, can be found at: https://www.youtube.com/watch?v=71RML96XxCI Below we provide full details on the actual dichoptic game play during training.

a. Establishing alignment, fusion, and perceptual matching adjustment before gameplay. Achieving adequate fusion and alignment is critical for amblyopic individuals when playing a dichoptic game. In order to establish and evaluate binocular fusion, participants completed an 'equalizer' procedure before the training intervention. At all times, participants viewed two images through a mirror stereoscope, from a viewing distance of $68 \mathrm{cms}$. First, each image was identical, except for the half crosses presented to each eye. The left eye viewed the bottom vertical and left arms while the right eye viewed the top vertical and right arms. With appropriate vergence, a whole cross with a blank square in its center was perceived. High-contrast surrounding frames and four squares were also presented to assist fusion see Ref. 54. While the subject viewed through the stereoscope, we decreased the contrast of the image presented to the strong eye until both frames were visible. Participants were then required to adjust the vertical and horizontal positions of the two frames separately until a full cross was perceived at the center of the image. Second, images of objects that were taken from the actual UT video game were presented, such that the AE and the strong eye each viewed one half-image. Participants were asked to adjust the alpha filter value of the strong eye until the object was perceived as having equal perceptual strength on both halves. This method of alpha blending (with a black background; see http://www.stonesc.com/pubs/Contrast\%20Metrics.htm for more details) alters the luminance and contrast of the complex images in the video game. A method-ofadjustment procedure was used to adjust the alpha filter level to the strong eye, so that the two eyes' images are reported as having equal perceptual strength in the two eyes. Participants viewed a total of 63 images: 7 different objects, each object appeared 9 times (each repetition with a different initial luminance value). The average value of the match points in these 63 'trials' was used as the initial alpha level to the strong eye for the game for each participant. By careful calibration, we converted the alpha levels to screen luminance values on our monitors, in order to calculate the interocular ratios (IORs). For participants unable to achieve alignment or fusion, the luminance of the strong eye was set to zero, having participants effectively playing monocularly for the initial few hours until binocular game play was feasible $(n=9)$.

b. Dichoptic gameplay. The video game was displayed on gamma corrected monitors, with screen resolution $1024 \times 768$ pixels and refresh rate $60 \mathrm{~Hz}$. At the beginning of every training session, participants were given trial frames with their refractive correction. Participants were asked to perform alignment and fusion by adjusting the stereoscope's mirrors before they started playing the game. Initial luminance level to the strong eye was set to be the same value used in the last session +50 on a scale between $0-255$ (or, for the first training session, the value obtained from the equalizer program). Participants saw the two frames with half cross in each, and were asked to adjust the stereoscope so that a full cross is perceived at the center (see description both cross halves are perceived as having equal luminance. This luminance value was used for that day's training session.

c. Progression of difficulty during gameplay. The goal of the game is to slay as many bots (computer controlled players or opponent) as possible without getting killed, within a certain time limit. Difficulty level of the game was altered either by increasing the number of bots or by increasing the bots' skill level (such as novice, average, experienced, expert etc.). Participants began the study by playing at an easy introductory level game map(s) for a total of 3 hours. These introductory maps introduce participants to the basics of game play, and contained fewer features compared to the regular maps. The number of bots in these introductory maps was increased gradually over these 3 hours (with 1, 3, 6,9 and 16 bots). The final introductory session, as well as all the remaining sessions of the game training with a given map, were played with 16 bots. During the training sessions, participants completed between three and five 23-minute 'blocks' of game play. In order to maintain a high level of interest, we chose three different 'Deathmatch' game maps to train participants. These maps were chosen such that they were similar in terms of visual appeal and sizes. Participants played each map for $\sim 12$ hours before switching to the next map. Whenever the training maps were switched, bot numbers were lowered to 9 for the first one or two blocks, and once subjects were familiar with the new map, the bot numbers were increased to 16. In each map, difficulty level (i.e. bot skill) was initially set to the easiest level, and was advanced to the next level if the ratio of kills to deaths was equal to or larger than $2: 1$ during the previous session.

d. Orientation discrimination task with Gabor target. An adaptive Gabor discrimination task, similar to the ones employed in perceptual learning studies, was presented to the AE during game play. A Gabor patch presented only to the $\mathrm{AE}$ (with Gaussian $\mathrm{SD}=1 \mathrm{deg}$ at $68 \mathrm{cms}$; visual angle of the carrier grating patch $\approx 4$ deg. per side), was tilted either to the right or the left $\left( \pm 35 \mathrm{deg}\right.$ from base angle $\left.90^{\circ}\right)$, and appeared every 6.9 seconds for a duration of 4.5 seconds. Participants were instructed to shoot the patch if it was tilted to the right, and to ignore it (or hit ' $\mathrm{E}$ ') if it was tilted to the left. If participants failed (either failed hitting right-tilted patches or incorrectly shot left-tilted patches), the Gabor patch turned into a bot that could kill the player in the game. Participants were instructed to prioritize the Gabor patches over other game bots. The Gabor target appeared approximately 315 times per hour of game play, at a set distance from the player.

The initial spatial frequency of the Gabor patch for each session was set to be the average frequency of the carrier gratings of the last 5 trials from the previous session minus $1 \mathrm{cpd}$. For instance, if the average was $4 \mathrm{cpd}$, the next session began with a Gabor stimulus consisting of a $3 \mathrm{cpd}$ carrier grating. The spatial frequency of the Gabor patch was adapted using a 3-up 1-down procedure ${ }^{55}$. The spatial frequency ranged from 0.2 to $9 \mathrm{cpd}$, and the carrier grating frequency increased by $0.2 \mathrm{cpd}$ after three consecutive correct responses. Normally sighted subjects who were piloted on the game reliably perceived up to $9 \mathrm{cpd}$ Gabors during gaming. This may be because resolution is decreased in a cluttered background with competing objects of interest ${ }^{5,57}$. Only one amblyope reached the $9 \mathrm{cpd}$ ceiling in the course of game play, which may have resulted in a slight underestimate of that participant's final resolution limit.

e. Additional Suppression checks during gameplay. In order to minimize the chances of suppression during dichoptic game play, we implemented frequent suppression checks. We presented a green crosshair to one eye, and a red crosshair to the other. Participants were instructed to make sure both crosshairs were visible and aligned during gameplay. If they failed to perceive one of the crosshairs, small luminance adjustments to the strong eye were made until both cross hairs were visible. This suppression check was in addition to ones described earlier: (a) setting the alignment and luminance level of strong eye at the beginning of every session and (b) the Gabor task: since the Gabor patches were presented only to $\mathrm{AE}$, the patch would be perceived only if the $\mathrm{AE}$ was not suppressed during dichoptic game play.

Visual Acuity and Stereopsis measurement. Visual acuity was measured using a high contrast ETDRS format chart comprised of Sloan optotypes (catalog No. 2104; Precision Vision, La Salle, Illinois). Right eye, left eye, and binocular acuity measurements were made at a 3 meter viewing distance. Participants wore best refractive correction, and the non-tested eye was patched during monocular measurements (results are summarized in Table 2).

We used the Randot Circles Stereotest (Stereo Optical Co., Inc.) to measure stereopsis. This is a vectographic test containing ten panels with stimulus disparity spanning 400-20 arc seconds. Each panel contained three contoured circles, of which only one had a crossed disparity. Subjects wore polarizing glasses and were asked to identify the circle that appeared to pop out of the plane (3-AFC task) from a $40 \mathrm{cms}$ viewing distance. Subjects began with the panel containing a 400 arc-second disparity test stimulus and continued down through the panels until they made an incorrect choice. If subjects gave an incorrect response, the preceding line was reassessed. Stereo-acuity was recorded as the disparity of the test stimulus in the last panel correctly labeled.

1. Levi, D. M. \& Li, R. W. Perceptual learning as a potential treatment for amblyopia: a mini-review. Vis. Res. 49, 2535-2549 (2009).

2. Li, R. W., Ngo, C., Nguyen, J. \& Levi, D. M. Video-game play induces plasticity in the visual system of adults with amblyopia. PLoS Biol. 9, e10011, 35 (2011). 
3. Jeon, S. T., Maurer, D. \& Lewis, T. L. The effect of video game training on the vision of adults with bilateral deprivation amblyopia. Seeing \& Perceiving. 25, 493-520 (2012).

4. Worth, C. A. \& Chevasse, F. B. Squint: Its Causes, Pathology and Treatment, $8^{\text {th }}$ Ed. (Blakiston, Philadelphia, 1950).

5. von Noorden, G. K. Binocular Vision and Ocular Motility. $2^{\text {nd }}$ Ed. (C.V. Mosby Co., St. Louis, 1980).

6. Levi, D. M., Harwerth, R. S. \& Smith, E. L. Humans deprived of normal binocular vision have binocular interactions tuned to size and orientation. Science 206, 852-854 (1979).

7. Levi, D. M., Harwerth, R. S. \& Smith, E. L. Binocular interactions in normal and anomalous binocular vision. Doc. Ophthalmol. 49, 303-324 (1980).

8. Smith, E. L. 3rd, Levi, D. M., Manny, R. E., Harwerth, R. S. \& White, J. M. The relationship between binocular rivalry and strabismic suppression. Invest. Ophthalmol. Vis. Sci. 26, 80-87 (1985).

9. Hess, R. F. The site and nature of suppression in squint amblyopia. Vis. Res. 31, 111-117 (1991).

10. Harrad, R. A. \& Hess, R. F. Binocular integration of contrast information in amblyopia. Vis. Res. 32, 2135-2150 (1992).

11. Baker, D. H., Meese, T. S. \& Hess, R. F. Contrast masking in strabismic amblyopia: attenuation, noise, interocular suppression and binocular summation. Vis. Res. 48, 1625-1640 (2008).

12. Maehara, G., Thompson, B., Mansouri, B., Farivar, R. \& Hess, R. F. The perceptual consequences of interocular suppression in amblyopia. Invest. Ophthalmol. Vis. Sci. 52, 9011-9017 (2011).

13. Mansouri, B., Thompson, B. \& Hess, R. F. Measurement of suprathreshold binocular interactions in amblyopia. Vis. Res. 48, 2775-2784 (2008).

14. Ding, J., Klein, S. A. \& Levi, D. M. Binocular combination in abnormal binocular vision. J Vis. 13(2), 14 (2013).

15. Ding, J. \& Levi, D. M. Rebalancing binocular vision in amblyopia. Ophthalmic Physiol. Opt. 34, 199-213 (2014).

16. Hess, R. F., Thompson, B. \& Baker, D. H. Binocular vision in amblyopia: structure, suppression and plasticity. Ophthal. Physiol. Opt. 34, 146-62 (2014).

17. Harrad, R., Sengpiel, F. \& Blakemore, C. Physiology of suppression in strabismic amblyopia. Br. J. Ophthalmol. 80, 373-377 (1996).

18. Sengpiel, F. \& Blakemore, C. The neural basis of suppression and amblyopia in strabismus. Eye 10, 250-258 (1996).

19. Bi, H., Zhang, B., Tao, X., Harwerth, R. S., Smith, E. L. \& Chino, Y. M. Neuronal responses in visual area V2 (V2) of macaque monkeys with strabismic amblyopia. Cereb. Cortex 21, 2033-2045 (2011).

20. Revell, M. J. Strabismus: A History of Orthoptic Techniques. (Barrie and Jenkins, London, 1971)

21. Ciuffreda, K. J., Levi, D. M. \& Selenow, A. Amblyopia: Basic and Clinical Aspects. (Butterworth-Heinemann, Stoneham, MA, 1991)

22. Knox, P. J., Simmers, A. J., Gray, L. S. \& Cleary, M. An exploratory study: prolonged periods of binocular stimulation can provide an effective treatment for childhood amblyopia. Invest. Ophthalmol. Vis. Sci. 53, 817-824 (2012).

23. Birch, E. E. Amblyopia and binocular vision. Prog. Ret. Eye Res. 33, 67-84 (2013).

24. Gambacorta, C., Huang, S., Vedamurthy, I., Nahum, M., Bayliss, J., Bavelier, D. \& Levi, D. Action Video Games as a Treatment of Amblyopia in Children: A Pilot Study of a novel, child-friendly action game. J. Vis. 14, 665; doi:10.1167/14.10.665 (2014)

25. Hess, R. F., Mansouri, B. \& Thompson, B. A new binocular approach to the treatment of amblyopia in adults well beyond the critical period of visual development. Restor. Neurol. Neurosci. 28, 793-802 (2010).

26. Hess, R. F., Mansouri, B. \& Thompson, B. A binocular approach to treating amblyopia: antisuppression therapy. Optom. Vis. Sci. 87, 697-704 (2010).

27. Hess, R. F., Mansouri, B. \& Thompson, B. Restoration of binocular vision in amblyopia. Strabismus 19, 110-118 (2011)

28. Black, J. M., Thompson, B. Maehara, G. \& Hess, R. F. A compact clinical instrument for quantifying suppression. Optom. Vis. Sci. 88, E334-343 (2011).

29. Li, J., Thompson, B., Deng, D., Chan, L. Y., Yu, M. \& Hess, R. F. Dichoptic training enables the adult amblyopic brain to learn. Curr Biol. 23, R308-309 (2013).

30. Ooi, T. L., Su, Y. R., Natale, D. M. \& He, Z. J. A push-pull treatment for strengthening the 'lazy eye' in amblyopia. Curr. Biol. 23, R309-310 (2013).

31. Barrett, B. T., Panesar, G. K., Scally, A. J. \& Pacey, I. E. A limited role for suppression in the central field of individuals with strabismic amblyopia. PLoS One 7, e36611 (2012).

32. Holopigian, K., Blake, R. \& Greenwald, M. J. Clinical suppression and amblyopia Invest. Ophthalmol. Vis. Sci. 29, 444-451 (1988).

33. Wong, A. M. Implications of interocular suppression in the treatment of amblyopia. J. AAPOS 15, 417-418 (2011)

34. Zhou, J., Jia, W., Huang, C. B. \& Hess, R. F. The effect of unilateral mean luminance on binocular combination in normal and amblyopic vision. Sci. Rep. 3 , 2012 (2013).

35. Zhang, P., Bobier, W., Thompson, B. \& Hess, R. F. Binocular Balance in Normal Vision and Its Modulation by Mean Luminance. Optom. \& Vis. Sci. 88, 1072-9 (2011)

36. Leonards, U. \& Sireteanu, R. Interocular suppression in normal and amblyopic subjects: the effect of unilateral attenuation with neutral density filters. Percept. \& Psychophys. 54, 65-74 (1993).
37. Rees, G., Kreiman, G. \& Koch, C. Neural correlates of consciousness in humans. Nat Rev Neurosci. 3, 261-70 (2002).

38. Wolfe, J. M. Stereopsis and binocular rivalry. Psychol Rev. 93, 269-82 (1986)

39. McKee, S. P., Levi, D. M. \& Movshon, J. A. The pattern of visual deficits in amblyopia. J. Vis. 3, 380-405, doi:10.1167/3.5.5 (2003).

40. Bonneh, Y. S., Sagi, D. \& Polat, U. Local and non-local deficits in amblyopia: acuity and spatial interactions. Vis. Res. 44, 3099-3110, doi:10.1016/j.visres.2004.07.031 (2004).

41. Song, S., Levi, D. M. \& Pelli, D. G. A double dissociation of the acuity and crowding limits to letter identification, and the promise of improved visual screening. J. Vis. 14, 3, doi:10.1167/14.5.3 (2014).

42. Levi, D. M. Prentice award lecture 2011: removing the brakes on plasticity in the amblyopic brain. Optom. Vis. Sci. 89, 827-838, doi:10.1097/ OPX.0b013e318257a187 (2012).

43. Polat, U. Restoration of underdeveloped cortical functions: evidence from treatment of adult amblyopia. Rest. Neurol. and Neurosci. 26, 413-424 (2008).

44. Polat, U. Making perceptual learning practical to improve visual functions. Vis. Res. 49, 2566-2573, doi:10.1016/j.visres.2009.06.005 (2009).

45. Li, R. W. \& Levi, D. M. Characterizing the mechanisms of improvement for position discrimination in adult amblyopia. J. Vis. 4, 476-487, doi:10,1167/4.6.7 (2004).

46. Li, R. W., Provost, A. \& Levi, D. M. Extended perceptual learning results in substantial recovery of positional acuity and visual acuity in juvenile amblyopia. Invest. Ophthalmol. Vis. Sci. 48, 5046-5051 (2007).

47. Zhang, J. Y., Cong, L. J., Klein, S. A., Levi, D. M. \& Yu, C. Perceptual learning improves adult amblyopic vision through rule-based cognitive compensation. Invest. Ophthalmol. Vis. Sci. 55, 2020-2030, doi:10.1167/iovs.13-13739 (2014).

48. Levi, D. M., Knill, D. C. \& Bavelier, D. Stereopsis and amblyopia: A mini-review. Vis. Res. doi.org/10.1016/j.visres.2015.01.002.

49. Bavelier, D., Levi, D. M., Li, R. W., Dan, Y. \& Hensch, T. K. Removing brakes on adult brain plasticity: from molecular to behavioral interventions. J. Neurosci. $\mathbf{3 0}$, 14964-71 (2010).

50. Epic Games. Unreal tournament, 2004. http://www.unrealtournament.com/ utgoty/, Accessed 2 November 2004.

51. Green, C. S. \& Bavelier, D. Action video game modifies visual selective attention. Nat. 423, 534-537 (2003)

52. Li, R., Polat, U., Makous, W. \& Bavelier, D. Enhancing the contrast sensitivity function through action video game training. Nat. Neurosci. 12, 549-551 (2009)

53. Bayliss, J. D., Vedamurthy, I., Nahum, M., Levi, D. M. \& Bavelier, D. Lazy Eye Shooter: a Novel Game Therapy for Visual Recovery in Adult Amblyopia. Paper presented at the IEEE International Games Innovation conference. DOI, 10.1109/ IGIC.2012.6329836 (2012).

54. Ding, J. \& Levi, D. M. Recovery of stereopsis through perceptual learning in human adults with abnormal binocular vision. Proc. Nat. Acad. Sci. (USA) 108, E733-741 (2011).

55. Levitt, H. Transformed up-down methods in psychoacoustics. J. Acoustic. Soc. Am. 49, Suppl 2, 467-76 (1971).

56. Bouma, H. Interaction effects in parafoveal letter recognition. Nat. 226, 177-178 (1970).

57. Levi, D. M. Crowding-an essential bottleneck for object recognition: a minireview. Vis. Res. 48, 635-654 (2008).

\section{Acknowledgments}

This research was supported by grant RO1EY020976 from the National Eye Institute and by the McDonnell Foundation "Critical Periods Network". We thank Jessica Bayliss for her role in game development.

\section{Author contributions}

These authors contributed to the study design and conceptualization: D.B. and D.L. These authors contributed to game development: I.V., D.B. and D.L. These authors contributed to piloting and fine-tuning, running the study and data analysis: I.V. and M.N. All authors contributed to writing.

\section{Additional information}

Supplementary information accompanies this paper at http://www.nature.com/ scientificreports

Competing financial interests: The authors declare no competing financial interests

How to cite this article: Vedamurthy, I., Nahum, M., Bavelier, D. \& Levi, D.M. Mechanisms of recovery of visual function in adult amblyopia through a tailored action video game. $\mathrm{Sci}$. Rep. 5, 8482; DOI:10.1038/srep08482 (2015).

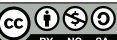

This work is licensed under a Creative Commons Attribution-NonCommercialShareAlike 4.0 International License. The images or other third party material in this article are included in the article's Creative Commons license, unless indicated otherwise in the credit line; if the material is not included under the Creative Commons license, users will need to obtain permission from the license holder in order to reproduce the material. To view a copy of this license, visit http:// creativecommons.org/licenses/by-nc-sa/4.0 